\title{
Spatiotemporal modelling and mapping of cervical cancer incidence among HIV positive women in South Africa: a nationwide study
}

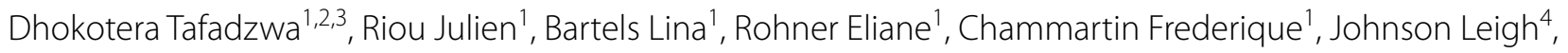
Singh Elvira ${ }^{2,5}$, Olago Victor ${ }^{2,5}$, Sengayi-Muchengeti Mazvita ${ }^{2,5}$, Egger Matthias ${ }^{1,4,6}$, Bohlius Julia ${ }^{1}$ and Konstantinoudis Garyfallos ${ }^{1,7^{*}}$ (D)

\begin{abstract}
Background: Disparities in invasive cervical cancer (ICC) incidence exist globally, particularly in HIV positive women who are at elevated risk compared to HIV negative women. We aimed to determine the spatial, temporal, and spatiotemporal incidence of ICC and the potential risk factors among HIV positive women in South Africa.

Methods: We included ICC cases in women diagnosed with HIV from the South African HIV cancer match study during 2004-2014. We used the Thembisa model, a mathematical model of the South African HIV epidemic to estimate women diagnosed with HIV per municipality, age group and calendar year. We fitted Bayesian hierarchical models, using a reparameterization of the Besag-York-Mollié to capture spatial autocorrelation, to estimate the spatiotemporal distribution of ICC incidence among women diagnosed with HIV. We also examined the association of deprivation, access to health (using the number of health facilities per municipality) and urbanicity with ICC incidence. We corrected our estimates to account for ICC case underascertainment, missing data and data errors.

Results: We included 17,821 ICC cases and demonstrated a decreasing trend in ICC incidence, from 306 to 312 in 2004 and from 160 to 191 in 2014 per 100,000 person-years across all municipalities and corrections. The spatial relative rate (RR) ranged from 0.27 to 4.43 in the model without any covariates. In the model adjusting for covariates, the most affluent municipalities had a RR of 3.18 (95\% Credible Interval 1.82, 5.57) compared to the least affluent ones, and municipalities with better access to health care had a RR of $1.52(1.03,2.27)$ compared to municipalities with worse access to health.
\end{abstract}

Conclusions: The results show an increased incidence of cervical cancer in affluent municipalities and in those with more health facilities. This is likely driven by better access to health care in more affluent areas. More efforts should be made to ensure equitable access to health services, including mitigating physical barriers, such as transportation to health centres and strengthening of screening programmes.

*Correspondence: g.konstantinoudis@imperial.ac.uk

${ }^{1}$ Institute of Social and Preventive Medicine (ISPM), University of Bern, Bern, Switzerland

Full list of author information is available at the end of the article

\section{Background}

HIV positive women have an up to five times higher risk of developing invasive cervical cancer (ICC) compared with HIV negative women [1]. Persistent infection with high-risk variants of the human papillomavirus (HPV) is a necessary cause for ICC [2]. In 2020, Southern Africa and Eastern Africa had the highest burden of cervical

c) The Author(s) 2021. This article is licensed under a Creative Commons Attribution 4.0 International License, which permits use, sharing, adaptation, distribution and reproduction in any medium or format, as long as you give appropriate credit to the original author(s) and the source, provide a link to the Creative Commons licence, and indicate if changes were made. The images or other third party material in this article are included in the article's Creative Commons licence, unless indicated otherwise in a credit line to the material. If material is not included in the article's Creative Commons licence and your intended use is not permitted by statutory regulation or exceeds the permitted use, you will need to obtain permission directly from the copyright holder. To view a copy of this licence, visit http://creativeco mmons.org/licenses/by/4.0/. The Creative Commons Public Domain Dedication waiver (http://creativecommons.org/publicdomain/ zero/1.0/) applies to the data made available in this article, unless otherwise stated in a credit line to the data. 
cancer worldwide with an incidence rate of over 40 and 36 cases per 100,000 population per year respectively [3, 4].

The two regions also have a high burden of HIV: 54\% of people living with HIV worldwide live in Southern and Eastern Africa [5]. A study looking at the global ICC estimated determine that in Southern Africa, 53\% of ICC cases were attributable to HIV [6].

In 2019, the World Health Organization called for the elimination of cervical cancer as a public health threat [7-9]. ICC can be prevented through HPV vaccination and early detection and treatment of pre-cancerous cervical lesions. When implemented effectively, these methods should result in decreased disease burden. However, globally there are disparities in ICC burden, even amongst HIV positive women. A global study demonstrated that HIV positive women in South Africa had a substantially higher incidence of ICC compared to the rest of the world, even after the introduction of antiretroviral therapy (ART) [10]. Poor access to screening, long waiting times for treatment of pre-cancerous cervical lesions as well as the socioeconomic inequities that exist in the country contribute to the high burden of ICC in South Africa [11, 12].

Spatiotemporal analysis of ICC incidence among HIV positive women can be used for disease surveillance over time and space, and to identify potential causes of the unequal distribution of ICC incidence. In this nationwide study in South Africa during 2004-2014, we examine the spatial, temporal and spatiotemporal incidence of ICC among women diagnosed with HIV. We perform three different corrections on the incidence rates to account for under-ascertainment, missing data and data errors. We assess whether urbanicity, deprivation and number of health facilities in a municipality (proxy for access to health services) explained any geographical or spatiotemporal patterns.

\section{Methods}

\section{Study setting}

In 2016, South Africa was divided into 9 provinces and 213 municipalities (Additional file 1: Figures S5-6). The area of the municipalities varies from $828.1 \mathrm{~km}^{2}$ to $44,231.3 \mathrm{~km}^{2}$. The population in 2016 was 55.7 million, varying from 8895 to $4,949,347$ across the municipalities. The ethnic breakdown amongst South African women was $80.6 \%$ Black, $8.1 \%$ White, $8.9 \%$ Mixed race and $2.4 \%$ Asian [13].

\section{Study population}

We retrieved data on women diagnosed with ICC with an HIV positive status in South Africa for the period 2004-2014 from the South African HIV cancer match
(SAM) study [14]. The SAM study aims to estimate cancer incidence in people living with HIV. It uses probabilistic record linkage methods to build a national cohort of people living with HIV from HIV-related laboratory tests stored at the National Health Laboratory Service (NHLS) and ICC cancer cases recorded in the National Cancer Registry (NCR) [14]. The HIV data includes laboratory HIV diagnostic tests like enzyme-linked immunosorbent assay (ELISA), qualitative and quantitative polymerase chain reaction (PCR) as well as the Western Blot. In addition, the HIV data includes HIV laboratory monitoring tests such as CD4 cell counts and HIV RNA viral loads [14]. The NCR is a nationwide, pathology-based cancer registry in South Africa with compulsory cancer reporting of cancer cases by both private and public laboratories since 2011 [15]. The NHLS is the largest diagnostic pathology service in South Africa, providing health services to about $80 \%$ of the population of South Africa (almost entirely users of public health facilities) [15].

We included ICC cases diagnosed within 2 years prior to a first HIV laboratory test or later, assuming that women would have been HIV-positive on average for at least 2 years prior to the test. The NHLS collects addresses of facilities where HIV tests are performed. We geocoded these addresses using Google Maps and linked them with corresponding municipalities. We assumed that the municipality of residence is the municipality where the women received the HIV-related test result.

We estimated the number of person-years of women diagnosed with HIV in each municipality using the Thembisa model (version 4.3; https://www.thembisa.org/ downloads) [16]. Thembisa is a mathematical model of the South African HIV epidemic, which provides provincial counts of cases diagnosed with HIV, stratified by age and sex. Since the Thembisa model does not provide estimates by municipality, we applied weights (by age, year and municipality) using NHLS data on women diagnosed with HIV to obtain municipal HIV estimates (see Additional file 1: Text S1.1 for the weight calculation and Figure $\mathrm{S} 1$ for the exclusion criteria of the NHLS).

\section{Outcome}

The NCR classifies cancer diagnoses according to the International Classification of Diseases for Oncology third edition (ICD-O-3), excluding pre-cancerous lesions [17]. We included cervical cancers coded C53.0, C53.1, C53.8 and C53.9.

\section{Co-variates}

We examined socioeconomic differentials across space using a multiple deprivation rank as determined in 2011 [18]. The most deprived ward is given a rank of 1 . To adjust for differences in access to health care, we 
considered the urbanicity of the municipalities (urbanrural) as retrieved from the National Department of Health data dictionary which was last updated in 2019 (Additional file 1: Figure S2), and the number of health facilities per municipality 2004-2014 from NHLS, as a proxy for access to health services (Additional file 1: Figure S3) [19]. The health facility information in the NHLS dataset refers to primary and secondary care clinics and hospitals as well as regional and national hospitals from which the HIV test was requested. The spatial and spatiotemporal analysis was based on the addresses of these health facilities.

\section{Statistical methods}

We used Bayesian hierarchical models to investigate the spatial and spatiotemporal distribution of ICC incidence among women diagnosed with HIV in South Africa. We first performed model selection without incorporating any covariates. Briefly, we used a re-parameterisation of the Besag-York-Mollié model to model the spatial autocorrelation [20-22]. This reparameterization includes a standard deviation hyperparameter that controls the variation of the field and a mixing hyperparameter indicating the proportion of the field that is dominated by strong spatial variation (ICAR prior [20]) as opposed to overdispersion. Random walk (RW) processes of order one were used to estimate the temporal random effect (on yearly resolution) and the random effects for the different age groups $(15-19,20-24, \ldots, 75-79,>80$ years). We fitted a series of models taking all possible combinations of the aforementioned random effects and also considered a type I interaction (interaction between an unstructured temporal and spatial random effects) [23]. We used penalized complexity priors for all the hyperparametes (Additional file 1: Text S1.2) [21, 24]. To examine model performance and select the best performing model we derived the deviance information criterion (DIC), the Watanabe-Akaike information criterion (WAIC), and the mean logarithmic score (CPO) [25]. In a second step, we used the model that fitted best and adjusted for the selected covariates. The model of best fit was determined as the one that minimizes the DIC, WAIC and CPO. We categorised the deprivation rank and number of health facilities per municipality in deciles to allow flexible fits. Additional file 1: Text S1.2 provides further information about the model specification.

\section{Incidence corrections}

We report four different estimates of the ICC incidence among women diagnosed with HIV. The first incidence estimates ('no correction') are from the best fitting model (without covariates).
The first correction ('correction I') accounts for the potential diagnosis of HIV in the private sector. NCR includes cases diagnosed in the private and public sector. The correction assumes that if an NCR case is diagnosed in the private sector, then the women are likely to have received the HIV diagnosis and care in the private sector, too. Out of the total 57,161 ICC cases diagnosed during 2004-2014 in South Africa, 32,564 were not linked with an HIV case. However, out of the 32,564 , only 5,077 (16\%) were diagnosed in the private sector. We assumed that $(57,161-32,564) / 57,161=43 \%$ of these cases are HIV related. Thus, for correction I, we oversampled $5077 * 0.43=2,183$ cases (on average 1,596 remained after the inclusion criteria) from the linked ICC cases that were treated in the private sector. To account for the sampling uncertainty, we fitted the model 100 times and averaged over the samples using Bayesian model averaging [26].

For the second correction ('correction II'), we used information about Kaposi sarcoma (KS). KS is a rare cancer in HIV-negative people but the most common cancer in people living with HIV [27]. In the SAM study, $70 \%$ of the $23,046 \mathrm{KS}$ cases could be linked probabilistically to an HIV test. Out of the KS cases not linked, $1890(8.2 \%)$ were diagnosed in the private sector. If we assume that all KS cases diagnosed from 2004 to 2014 occurred among people living with HIV, then the remaining $30 \%-8.2 \%=21.8 \%$ missing HIV tests may be due to under-ascertainment of HIV, data errors or inaccuracies preventing linkage. To correct for the above, we calculated the proportion of unlinked KS cases by municipality excluding those treated in the private sector (because correction I accounts for that), and corrected the recorded numbers of ICC cases using the KS linked proportions (Additional file 1: Text S1.3). The third correction combines, by means of summing, the additional cases from corrections I and II (referred to as 'full correction').

We report median posterior and 95\% credibility intervals (CrIs) for the temporal ICC incidence rate and performed age standardisation based on the world population weights [28]. We report median posterior of the exponential of the spatial random effects (spatial relative rates or spatial RRs; relative to the national average incidence rate over time), RRs for the effect of the selected covariates (relative to the baseline) and posterior probabilities (that the RRs is higher than 1). We used integrated nested Laplace approximation (INLA) for Bayesian inference [29]. The data and the code used for the analysis are online available https://github.com/ gkonstantinoudis/CervixHIVRSA. 


\section{Results}

\section{Study setting and population}

We identified 57,161 ICC cases diagnosed from 2004 to 2014 in South Africa in the SAM study. We excluded 32,393 cases not linked with an HIV record and $\geq 15$ years old, 1607 with missing or imprecise geocodes, 3666 with a ICC diagnosis 2 years or more before their first HIV related laboratory test and 1674 cases residing in KwaZulu-Natal leaving us with 17,821 ( 32\%) cases for the main analysis (Fig. 1). We excluded the

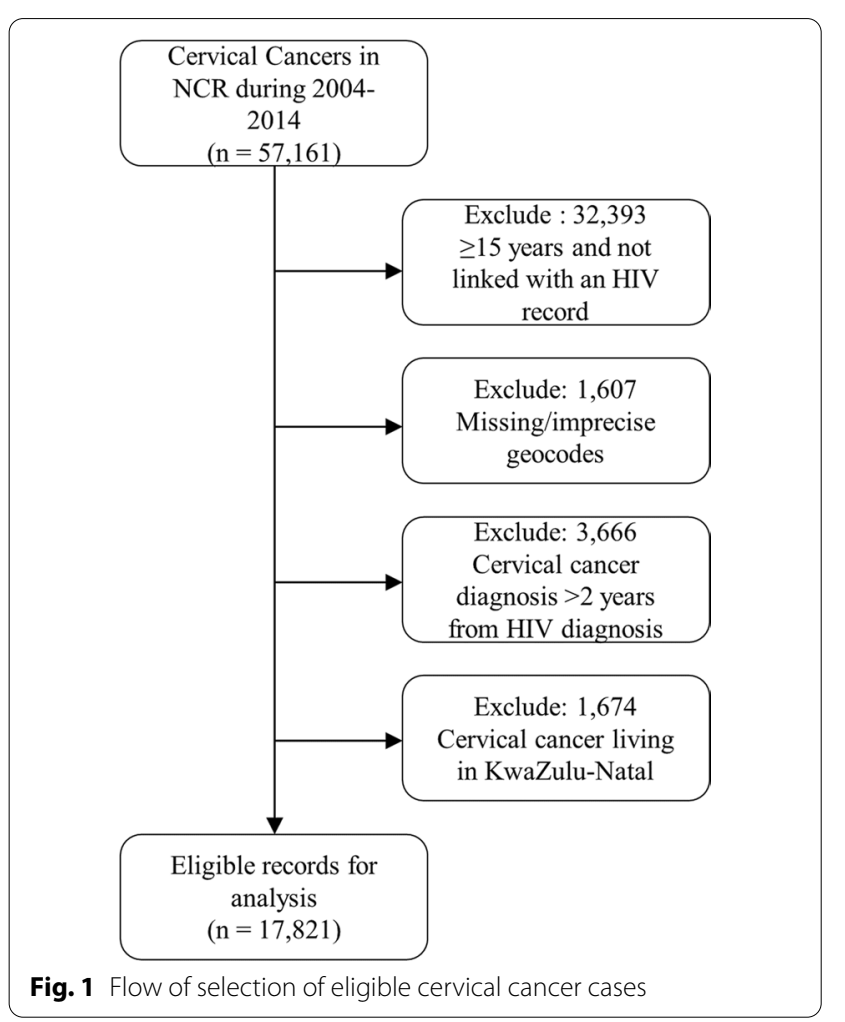

KwaZulu-Natal province because it started contributing data to NHLS only in 2010. With correction I, the total number of cases varied from 19,380 to 19,466 . It was 21,446 for correction II and varied from 23,005 to 23,091 for the full correction. The total person-years (PY) at risk among women diagnosed with HIV during 2004-2014 were $24,059,679$.

\section{Model selection}

Based on the DIC, WAIC and CPO the model with the best fit was the one with random effects for age, time, space and a space-time interaction (Additional file 1: Table S1).

\section{Co-variates}

The spatial distribution of the deciles of deprivation rank and number of health facilities is shown in Fig. 2. The spatial distribution of urbanicity is shown in the Additional file 1: Figure S2. There were 23 urban and 190 rural municipalities in South Africa in 2016. Overall, we observe substantial spatial variation for all three covariates.

\section{Overall incidence}

The median overall ICC incidence during 2004-2014 from the best fitting model was 294 (95\% CrI: 174, 498) for no correction, 297 (95\% CrI: 163, 573) for correction I, 310 (95\% CrI: 170, 494) for correction II and 312 $(95 \%$ CrI: 171, 510) for the full correction, per 100,000 PY, among women diagnosed with HIV (Additional file 1: Table S2).

\section{Temporal trends}

Figure 3 shows that the annual median age-standardized ICC incidence rate per 100,000 PY of women diagnosed with HIV in South Africa decreased during 2004-2014.

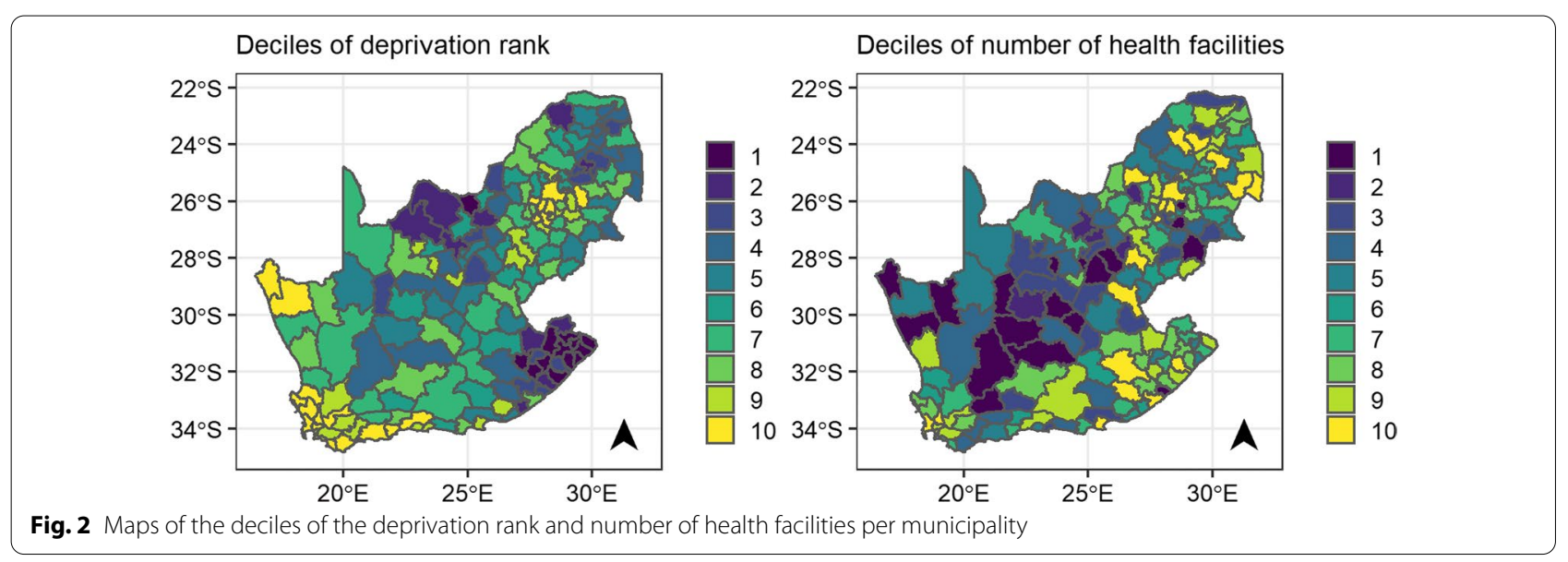



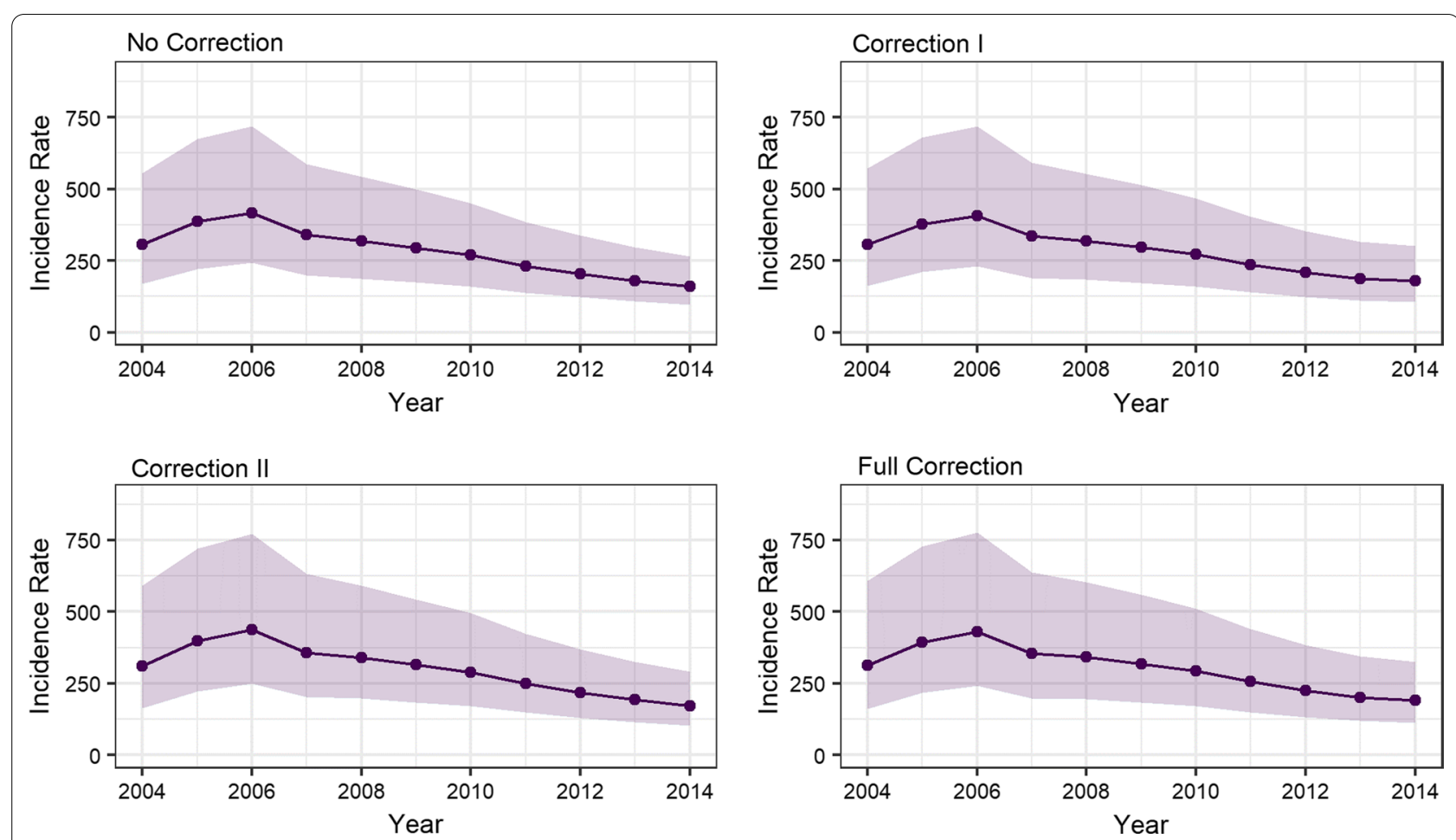

Fig. 3 Yearly age-standardised incidence rate per 100,000 person years for women living with HIV in South Africa

In 2004, the incidence rate varied from 306 (95\% CrI: 169, $555)$ for no correction, to 306 (95\% CrI: 163, 573) for correction I, and to 310 (95\% CrI: 164, 589) for correction II and 312 (95\% CrI: 160, 609) for the full correction, per 100,000 PY (Table S2 in Additional file 1:). In contrast, in 2014 the incidence rate varied from 160 (95\% CrI: 96, 265) for no correction, to 179 (95\% CrI: 106, 303) for correction I, to 172 (95\% CrI: 103, 290) for correction II and to 191 (95\% CrI: 113, 326) for the full correction, per 100,000 PY (in Additional file 1: Table S2).

\section{Spatial patterns}

Figure 4 shows the median posterior spatial RR and posterior probability of ICC across women diagnosed with HIV in the no correction model without covariates (top panels) and the no correction model adjusted for the selected covariates (bottom panels). For the model without covariates, the spatial RR ranged from 0.27 (95\% CrI: $0.16,0.42)$ to 4.43 (95\% CrI: $3.71,5.28)$. There was attenuation of associations when adjusting for covariates with the RR varying from 0.35 (95\% CrI: $0.18,0.64$ ) to 4.10 (95\% CrI: 2.90, 5.78). Nevertheless, the spatial variation remained strong after having adjusted for the selected covariates, with the mixing hyperparameter being 0.77 $(0.45,0.95)$, Additional file 1: Table S3. RR and posterior probabilities were larger in the province of the Northern Cape (north-western region of the map) and the
Limpopo, Gauteng and Mpumalanga provinces (northeastern part of the map) (Additional file 1: Figure S3). The results across the incidence corrections are similar, Additional file 1: Figure S7.

\section{Spatiotemporal patterns}

The spatial differences have not attenuated over the years, and the patterns do not seem to be consistent in both 'no correction' models, with and without covariates (Fig. 5 and Additional file 1: Figure S8). The results across the incidence corrections are similar, Additional file 1: Figure S9-15.

\section{Influence of covariates}

We assessed the influence of covariates in the no correction model (Table 1). The RR comparing the least with the most deprived municipalities was 3.56 (95\% CrI: 2.16-5.89) in the univariable model, and 3.18 (95\% CrI: $1.82-5.57)$ in the multivariable model. The RR in municipalities with the highest number of health facilities was 1.83 (95\% CrI: $1.27-2.64$ ) in the univariable model, and 1.52 (95\% CrI: 1.03-2.27) in the multivariate model, compared to municipalities with the lowest number of health facilities. There was little evidence of a difference in ICC incidence across urban and rural municipalities, both in the univariable $(1.19,95 \% \mathrm{CrI} 0.94-1.50)$ and multivariable model $(0.85$, 95\% CrI 0.65-1.12). 


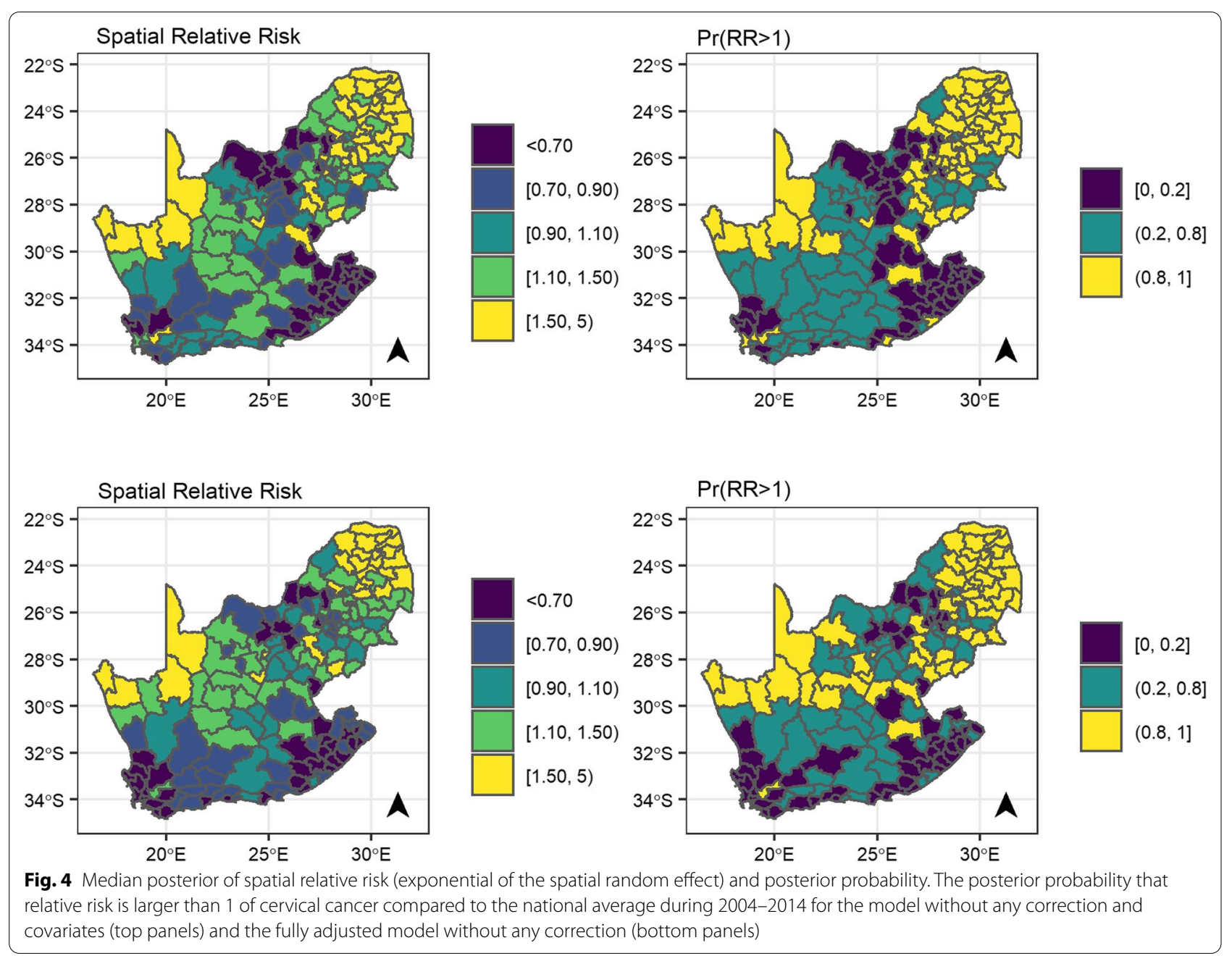

\section{Discussion}

\section{Main findings}

In this study, we determined the spatial, temporal and spatiotemporal variation of ICC incidence in women diagnosed with HIV in South Africa, and assessed whether urbanicity deprivation and number of health facilities explained any geographical or spatiotemporal patterns. We observed an overall decreasing trend in ICC incidence in women diagnosed with HIV in South Africa during 2004-2014. We found a higher relative incidence in the Northern Cape and in the Limpopo, Gauteng and Mpumalanga provinces. Regarding the spatiotemporal patterns, the spatial differences did not decrease over time. Also, HIV positive women residing in the least deprived municipalities or in municipalities with larger numbers of health facilities were more likely to get diagnosed with ICC.

\section{Discussion in the context of previous studies}

The incidence observed across all corrections is consistent with other studies on ICC incidence in women living with HIV in South Africa. Two hospital-based cohort studies determined crude incidence rates that ranged from 447 to 506 ICC cases/100,000 person-years [10, 30]. These results reflect the high ICC burden in HIV positive women in South Africa compared to the overall crude incidence rate of ICC in the general South African population. The latter experienced an incidence of 22.68 ICC cases/100,000 of the population as of 2016 [31]. Other $\mathrm{HIV}$ and cancer registry linkage studies in Africa have shown variable crude incidence rates in women living with HIV. In Nigeria, investigators observed an incidence of 7.8/ 100,000 person-years [32] whilst in Uganda, the incidence reported was 70/ 100000 person-years, with the incidence being highest in the 35-44 age group at 


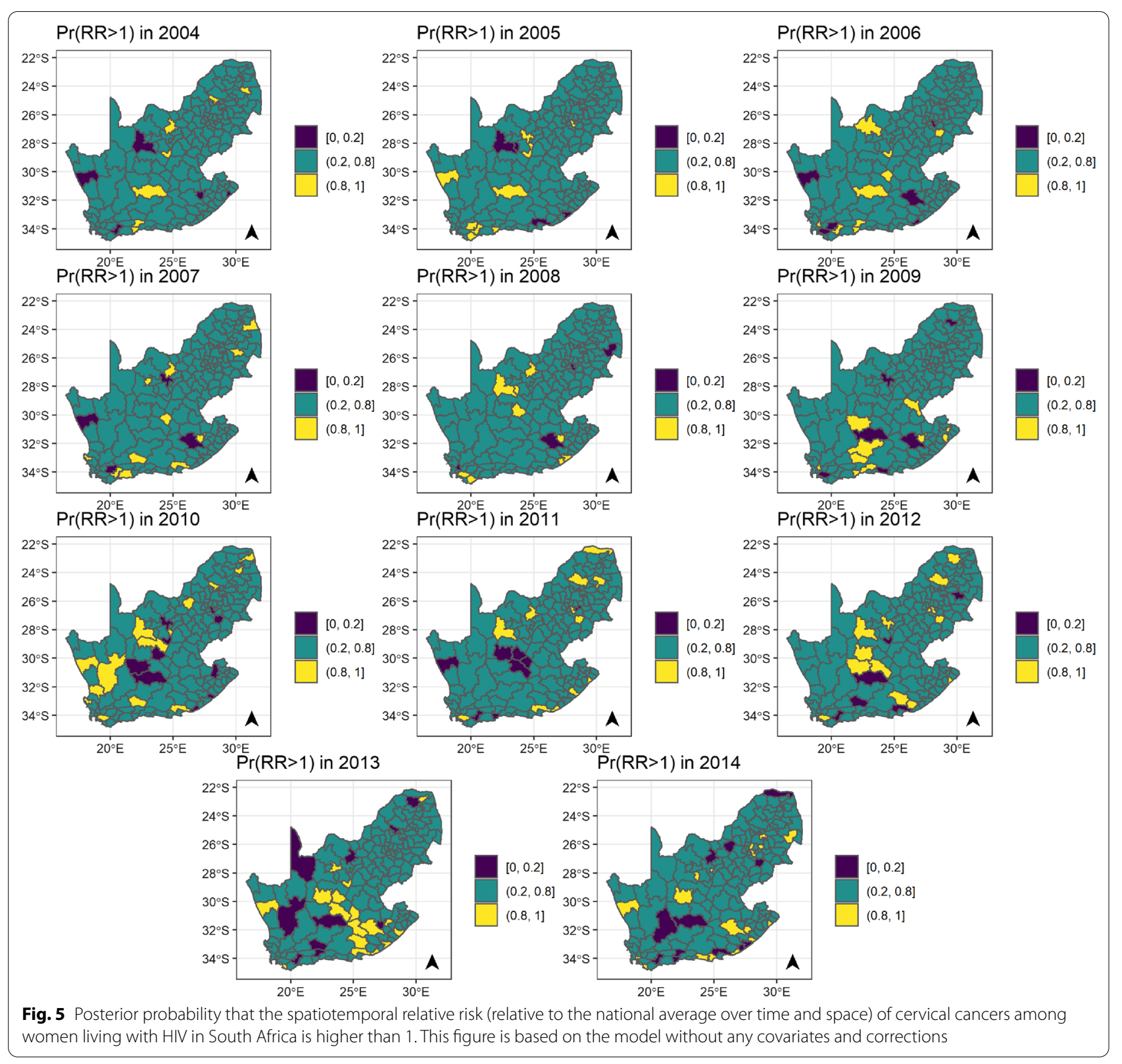

200/100,000 person-years [33]. These differences in ICC incidence might potentially reflect differences in access to ICC screening and diagnosis in the different countries.

Although CC incidence in women diagnosed with HIV is still high compared to the general population, we observed a decreasing trend in ICC incidence after an initial increase between 2004 and 2006, similar to the decline that was observed in high income countries after the introduction of ART [34, 35]. However, in other African settings, the ICC incidence trends either remained unchanged or increased even after the introduction of ART $[33,36]$. The decline in incidence in our study might reflect the HIV testing patterns in South Africa across the study period. Initially, fewer people were tested for HIV [37] and those tested had an increased burden of disease and lower CD4 cell counts [38]. Increased ICC incidence has been associated with very low $\mathrm{CD} 4$ cell counts compared to higher CD4 cell counts. With time, as HIV testing expanded, more women had access to timely HIV diagnosis and treatment before advanced disease, thus reducing the likelihood of ICC. The timely access might have resulted in the subsequent decline in ICC incidence from 2006 amongst women diagnosed with HIV. ART reduces the prevalence of high-risk HPV infections and 
Table 1 Median posterior relative rate and 95\% credibility intervals of the effect of the covariates

\begin{tabular}{|c|c|c|c|c|c|c|c|}
\hline \multirow[b]{2}{*}{ Co-variates } & & \multicolumn{3}{|c|}{ Univariable models } & \multicolumn{3}{|c|}{ Mutlivariable model } \\
\hline & & Median & $95 \% \mathrm{Crl}$ & $\operatorname{Pr}(\mathrm{RR}>1)$ & Median & $95 \% \mathrm{Crl}$ & $\operatorname{Pr}(\mathrm{RR}>1)$ \\
\hline \multirow[t]{2}{*}{ Urbanicity } & Rural & 1.00 & - & 1.00 & 1.00 & - & 1.00 \\
\hline & Urban & 1.19 & $(0.94,1.50)$ & 0.92 & 0.85 & $(0.65,1.12)$ & 0.12 \\
\hline \multirow[t]{10}{*}{ Deprivation rank } & 1st Decile & 1.00 & - & 1.00 & 1.00 & - & 1.00 \\
\hline & 2nd Decile & 1.81 & $(1.16,2.82)$ & 1.00 & 1.77 & $(1.13,2.78)$ & 0.99 \\
\hline & 3rd Decile & 1.84 & $(1.19,2.86)$ & 1.00 & 1.68 & $(1.07,2.66)$ & 0.99 \\
\hline & 4th Decile & 2.14 & $(1.35,3.40)$ & 1.00 & 1.97 & $(1.23,3.16)$ & 1.00 \\
\hline & 5th Decile & 1.76 & $(1.09,2.83)$ & 0.99 & 1.83 & $(1.10,3.04)$ & 0.99 \\
\hline & 6th Decile & 2.22 & $(1.41,3.49)$ & 1.00 & 1.97 & $(1.23,3.17)$ & 1.00 \\
\hline & 7th Decile & 2.40 & $(1.50,3.85)$ & 1.00 & 2.33 & $(1.43,3.79)$ & 1.00 \\
\hline & 8th Decile & 2.82 & $(1.78,4.50)$ & 1.00 & 2.69 & $(1.66,4.35)$ & 1.00 \\
\hline & 9th Decile & 3.21 & $(1.98,5.21)$ & 1.00 & 2.88 & $(1.75,4.74)$ & 1.00 \\
\hline & 10th Decile & 3.56 & $(2.16,5.89)$ & 1.00 & 3.18 & $(1.82,5.57)$ & 1.00 \\
\hline \multirow[t]{10}{*}{ Number of health facilities } & 1st Decile & 1.00 & - & 1.00 & 1.00 & - & 1.00 \\
\hline & 2nd Decile & 1.16 & $(0.68,1.98)$ & 0.71 & 1.14 & $(0.67,1.92)$ & 0.69 \\
\hline & 3rd Decile & 0.97 & $(0.66,1.42)$ & 0.43 & 0.90 & $(0.62,1.31)$ & 0.29 \\
\hline & 4th Decile & 1.14 & $(0.77,1.68)$ & 0.74 & 1.04 & $(0.71,1.55)$ & 0.59 \\
\hline & 5th Decile & 1.24 & $(0.86,1.81)$ & 0.87 & 1.06 & $(0.73,1.54)$ & 0.61 \\
\hline & 6th Decile & 1.17 & $(0.79,1.74)$ & 0.78 & 1.07 & $(0.72,1.60)$ & 0.64 \\
\hline & 7th Decile & 1.15 & $(0.80,1.67)$ & 0.77 & 1.05 & $(0.72,1.56)$ & 0.61 \\
\hline & 8th Decile & 1.31 & $(0.91,1.89)$ & 0.93 & 1.18 & $(0.81,1.71)$ & 0.81 \\
\hline & 9th Decile & 1.35 & $(0.93,1.97)$ & 0.95 & 1.24 & $(0.85,1.83)$ & 0.87 \\
\hline & 10th Decile & 1.83 & $(1.27,2.64)$ & 1.00 & 1.52 & $(1.03,2.27)$ & 0.98 \\
\hline
\end{tabular}

$\mathrm{Crl}$, Credibility intervals; $\operatorname{Pr}(\mathrm{RR}>1)$, Posterior probability that the relative rate is larger than 1. The models were adjusted for urbanicity, deprivation rank and number of health facilities as a proxy for access to health

cervical pre-cancerous lesions. Still, the effect is reduced in individuals initiating ART at an advanced HIV stage and in women with advanced pre-cancerous lesions [39-41]. The expansion of the national ART programme, which started in 2004, ensured that more people had access to HIV treatment and subsequently improved CD4 cell counts. This expansion could also explain the subsequent decline in incidence from 2006 to 2014 as women had a lower burden of disease and better access to ART. However, in some studies this has not been supported. In Botswana, no significant change in ICC incidence was observed after ART expansion [36]. Spatial analyses have been used in ICC studies to determine disparities in incidence and mortality as well as the factors associated with the unequal distribution of ICC burden [30, 42-45]. However, all these studies focused on the general population regardless of HIV status. Previous studies examining the spatial trends in the USA, Thailand and Cuba have reported variations in ICC incidence driven by a variety of factors $[42,46,47]$. In Thailand, Zhao et al. observed differences in ICC incidence in districts in the Songhkla province driven by sexual behaviour and migration patterns [47]. A similar study in Cuba showed that differences in screening programme coverage did not account for the spatial differences in ICC incidence, which were better explained by differences in lifestyle and socioeconomic status [43]. In the USA, the disparities were attributed to ethnicity, socioeconomic status and screening coverage [42]. Low socioeconomic status of areas and low screening rates were associated with an increased ICC incidence. Another spatiotemporal study in Texas, USA demonstrated that poor education, African and Hispanic ethnicities and low socioeconomic status was associated with a high ICC incidence [48]. In South Africa, Black women have a higher incidence of ICC compared to other races. The incidence of ICC amongst black women in 2017 was 28.25 per 100000 women compared to 8.29, 13.66 and 15.24 in Asian, Mixed race and White women respectively [31]. This is largely attributed to differences in HIV prevalence as well as unequal access to health care services. However, in our study data on ethnicity was not available.

In our study, more affluent areas had a higher incidence of ICC. People living in the least deprived municipalities were about three times more likely to be diagnosed with ICC. This points towards disparities in 
ICC, driven by unequal access to services due to socioeconomic circumstances. In Uganda, a spatial analysis of ICC in women, regardless of HIV status, showed that areas of low socioeconomic status had decreased access to care, including ICC screening [44]. This can lead to a spurious negative association between ICC incidence and socioeconomic status, as areas of low socioeconomic status would appear to have a lower burden of disease when the ICC cases are not being diagnosed. Some districts in South Africa have pronounced shortages of health care staff, with multiple vacant posts and ill-equipped facilities [49], which affects access to care. In addition, utilisation of public health sector facilities in South Africa has been shown to be higher among people from least deprived areas, with individuals from deprived areas having limited access to public health sector services [50].

We observed a relationship between the number of facilities per municipality and ICC incidence in women diagnosed with HIV: the greater the number of health facilities, the higher the ICC incidence. In our study, we used the number of health facilities per municipality as a proxy for differences in access to health care services. This points to the effect of inequalities in access to care on ICC incidence. In the United States, in urban areas, a larger distance to the health facility was a barrier to accessing cervical cancer care and treatment [51]. However, the same was not observed in rural areas, where long distances were associated with better access, with the suggested reason being the familiarity and tolerance to long-distance travel for health services in rural areas [51]. A qualitative study in Botswana determined thematic issues that affected access to ICC treatment and care. Among those were contextual issues like distance from facility, other commitments and transportation issues as well as health system issues such as long waiting times for appointments with health care workers and for test results [52]. In South Africa, it is estimated that for every additional health care professional per year, the likelihood of cervical cancer screening improved by $1 \%$ [53].

Deprivation, access to health and urbanicity did not explain all of the observed spatial variation. The remaining spatial trends can point towards geographical health inequalities, potentially generated by different screening programmes and municipality level health policies. Differences in HPV prevalence might also influence the ICC incidence patterns. There was no readily available nationwide screening coverage data or ethnicity data at the municipality level for women diagnosed with HIV at the time of analysis.

\section{Strengths and limitations}

To our knowledge, our study is the first nationwide study to describe the temporal, spatial and spatiotemporal variation in ICC incidence by municipality amongst women diagnosed with HIV in South Africa. Given that our data sources cover the majority of the South African population, and given the incidence adjustments we made, our incidence estimates are expected to cover the majority of women diagnosed with HIV in South Africa. The correction factors reduced the selection bias introduced through our data sources and the record linkage process. However, our study also has several limitations. In our study, the population under observation was specific to a sub-population of women who knew their HIV status and thus had contact with health care facilities. This contact increases the probability of being referred for ICC screening and treatment when compared to women who are unaware of their HIV status. As a result, the results cannot be generalised to women who have not been diagnosed with HIV. According to a survey by the Human Science Research Council, $47.5 \%$ of women aged 15 years and older were unaware of their HIV positive status in 2017 [54]. Although we introduced several incidence corrections, women without health care access are missing from our study. The ecological nature of our analysis did not allow us to adjust for CD4 counts that might influence ICC incidence. We could not adjust for screening coverage due to the absence of nationwide screening data for women living with HIV. While disparities in ICC incidence by ethnicity have been shown in South Africa, we lacked information on ethnicity in our data which might have explained some of the differences in ICC across municipalities [31]. Prior to 2011, NCR consisted mainly of cancer cases reported by public laboratories. In 2011, compulsory cancer reporting was established by both private and public laboratories $[15,55]$. This is not expected to have substantially affected ICC reporting in HIV-positive women, since almost all HIV-positive women receive treatment through public sector services. Lastly, in our study, we could not assess any of the aforenmentioned different metrics pertaining to access. We should also note that we assumed that the municipality of the health facility is the same as the municipality of residence. This assumption may be problematic as people can move to other provinces and municipalities for healthcare. For instance, municipalities with more facilities might have better accessibility and shorter waiting times and hence people might choose to go there instead. Thus, we cannot rule out that the observed effect can be driven by this assumption. 


\section{Conclusion}

In conclusion, although the burden of ICC among women diagnosed with HIV has decreased over time, there was strong spatial variation. Individuals from less deprived areas and areas with more health facilities are more likely to be diagnosed with ICC compared to those from most deprived areas or areas with fewer health facilities. The geographical discrepancies persisted after adjusting for the aforementioned factors, which could reflect spatial differences in screening policies across women living with HIV. More efforts should be made to ensure equitable access to health services, including mitigating physical barriers, such as transportation to health centres, strengthening of screening programmes, and nationwide HPV vaccination implementation in populations living with HIV. Special attention should be paid to women living in low socioeconomic areas.

\begin{abstract}
Abbreviations
ART: Antiretroviral therapy; CPO: Mean logarithmic score; Crl: Credibility Intervals; DIC: Deviance Information Criterion; ELISA: Enzyme-linked immunosorbent assay; HPV: Human Papillomavirus; ICAR: Intrinsic conditional autoregressive process; ICC: Invasive cervical cancer; ICD-O-3: International Classification of Diseases for Oncology third edition; INLA: Integrated Nested Laplace approximation; KS: Kaposi sarcoma; NCR: National Cancer Registry; NHLS: National Health Laboratory Service; PCR: Polymerase Chain Reaction; PY: Person Years; RW: Random Walk; RR: Relative rates; SAM: South African HIV cancer match; WAIC: Watanabe-Akaike Information Criterion.
\end{abstract}

\section{Supplementary Information}

The online version contains supplementary material available at https://doi. org/10.1186/s12942-021-00283-z.

Additional file 1: Table S1. Deviance information criterion (DIC), Watanabe-Akaike information criterion (WAIC) and mean logarithmic score (CPO) for the different models considered. For the notation. Table S2. Annual median and 95\% Credibility intervals (Crl) for the incidence rate of cervical ancers among HIV positive women per 100,000 person years in South Africa for the different corrections considered. Table S3. Results of the model with spatial, temporal and spatiotemporal interaction and depri-vation and urbanicity for the different Thembisa denominators. Figure S1. Flowchart for the exclusion criteria used to calculate weights using data from the Na-tional Health Laboratory Service (NHLS) to disaggregate the Thembisa provincial es-timates. Figure $\mathbf{S 2}$. The spatial variation of urbanicity (urban/rural) in South Africa. Figure S3. The number of health facilities by municipality and year (left panel) and by municipality in 2014 (right panel). Figure S4. The spatial variation of socioeconomic index in South Africa. A rank of 1 denotes the most deprived area. Figure S5. Provinces in South Africa in 2016 [ROSEA]. Figure S6. Municipalities in South Africa in 2016 [ROSEA]. Figure S7. Median posterior of spatial relative risk (exponential of the spatial random effect) and posterior probability using correction I, II and full correction. Figure S8. Posterior probability that the spatiotemporal relative risk (relative to the national av-erage over time) is higher than 1 of cervical cancers among women living with HIV in South Africa, using the no correction model adjusted for the selected covariates.

\section{Acknowledgements}

The authors would like to thank all funders, the National Health Laboratory Service (NHLS), the NHLS's Corporate Data Warehouse and the National Cancer Registry.

\section{Authors' contributions}

Conceptualisation: KG, EM, SE and BJ; data acquisition: MM, SE and JL (Thembisa estimates). Methodology: BL and OV were involved in data cleaning and conducted record linkages; formal analysis and validation: KG; writing-original draft: KG and DT. All authors contributed towards data interpretation and critical comments on the first and/or subsequent drafts. All authors read and approved the final manuscript.

\section{Funding}

Research reported in this publication was supported by the Eunice Kennedy Shriver National Institute Of Child Health \& Human Development (NICHD), National Institute Of Diabetes And Digestive And Kidney Diseases (NIDDK), National Institute On Drug Abuse (NIDA), National Heart, Lung, And Blood Institute (NHLBI), National Institute On Alcohol Abuse And Alcoholism (NIAAA), Fogarty International Center (FIC), National Cancer Institute (NCI), and by the National Institute of Allergy and Infectious Diseases of the National Institutes of Health under Award Number U01 Al069924. The content is solely the responsibility of the authors and does not necessarily represent the official views of the National Institutes of Health. Research reported in this publication was supported by the National Institutes of Health administrative supplement to Existing NIH Grants and Cooperative Agreements (Parent Admin Supp) (The South African HIV Cancer Match Study; U01AI069924-09, PI Matthias Egger, co-PI Julia Bohlius) PEPFAR supplement (PI Matthias Egger), the Swiss National Science Foundation (The South African HIV Cancer Match Study, 320030_169967, PI Julia Bohlius) and the U.S. CRDF Global (Beginning Investigator Grant for Catalytic Research in Cancer, Burden of Cancers Associated with HIV_DAA3-16-62705-1, PI Mazvita Sengayi), and allocated for this project. The contents are solely the responsibility of the authors and do not necessarily reflect the views of the funding bodies. Garyfallos Konstantinoudis is supported by an MRC Skills Development Fellowship [MR/T025352/1]. This project has received funding from the European Union's Horizon 2020 research and innovation programme under the Marie Skłodowska-Curie grant agreement No 801076, through the SSPH + Global PhD Fellowship Programme in Public Health Sciences (GlobalP3HS) of the Swiss School of Public Health (PhD Candidate: Tafadzwa Dhokotera).

\section{Availability of data and materials}

The aggregated data will be made available at https://github.com/gkonstanti noudis/CervixHIVRSA.

\section{Declarations}

Ethics approval and consent to participate

Permission to use the routinely collected NHLS and NCR data was sought from the relevant authorities. Ethical approval to conduct the study was granted by the University of the Witwatersrand Human Research Ethics Committee [Ethics certificate numbers (SAM: M160944)].

\section{Consent for publication}

Both the NCR and the NHLS gave consent to publish the routinely collected data.

\section{Competing interests}

The authors declare no competing interests.

\section{Author details}

${ }^{1}$ Institute of Social and Preventive Medicine (ISPM), University of Bern, Bern, Switzerland. ${ }^{2}$ National Cancer Registry, National Health Laboratory Service, Johannesburg, South Africa. ${ }^{3}$ Graduate School for Cellular and Biomedical Sciences, University of Bern, Bern, Switzerland. ${ }^{4}$ Centre for Infectious Disease Epidemiology and Research, School of Public Health and Family Medicine, University of Cape Town, Cape Town, South Africa. ${ }^{5}$ Division of Epidemiology and Biostatistics, School of Public Health, University of the Witwatersrand, Johannesburg, South Africa. ${ }^{6}$ Population Health Sciences, Bristol Medical School, University of Bristol, Bristol, UK. ${ }^{7}$ Epidemiology and Biostatistics Department, School of Public Health, Imperial College London, London, UK.

Received: 23 February 2021 Accepted: 15 June 2021

Published online: 29 June 2021 


\section{References}

1. Ghebre RG, Grover S, Xu MJ, Chuang LT, Simonds H. Cervical cancer control in HIV-infected women: past, present and future. Gynecol Oncol Reports. 2017;21:101-8.

2. Bouvard $V$, Baan $R$, Straif $K$, et al. A review of human carcinogens - part B: biological agents. Lancet Oncol. 2009;10:321-2.

3. Bray F, Ferlay J, Soerjomataram I, Siegel RL, Torre LA, Jemal A. Global cancer statistics 2018: GLOBOCAN estimates of incidence and mortality worldwide for 36 cancers in 185 countries. CA Cancer J Clin. 2018;68:394-424

4. Sung H, Ferlay J, Siegel RL, et al. Global cancer statistics 2020: GLOBOCAN estimates of incidence and mortality worldwide for 36 cancers in 185 countries. CA Cancer J Clin. 2021;71(3):209-49.

5. UNAIDS. 2018 Global HIV Statistics. Unaids 2019; pp. 1-6. https://www. avert.org/professionals/hiv-around-world/sub-saharan-africa/overview.

6. Stelzle D, Tanaka LF, Lee KK, et al. Estimates of the global burden of cervical cancer associated with HIV. Lancet Glob Heal. 2021;9:e161-9.

7. Brisson M, Drolet M. Global elimination of cervical cancer as a public health problem. Lancet Oncol. 2019;20:319-21.

8. Simms KT, Steinberg J, Caruana M, et al. Impact of scaled up human papillomavirus vaccination and cervical screening and the potential for global elimination of cervical cancer in 181 countries, 2020-99: a modelling study. Lancet Oncol. 2019;20:394-407.

9. Hall MT, Simms KT, Bin LJ, et al. The projected timeframe until cervical cancer elimination in Australia: a modelling study. Lancet Public Heal. 2019;4:e19-27.

10. Rohner E, Sengayi M, Goeieman B, et al. Cervical cancer risk and impact of Pap-based screening in HIV-positive women on antiretroviral therapy in Johannesburg, South Africa. Int J Cancer. 2017;141:488-96.

11. Olorunfemi G, Ndlovu N, Masukume G, Chikandiwa A, Pisa PT, Singh E. Temporal trends in the epidemiology of cervical cancer in South Africa (1994-2012). Int J Cancer. 2018;143:2238-49.

12. Jordaan S, Michelow P, Simoens C, Bogers J. Challenges and progress of policies on cervical cancer in South Africa. Heal Care Curr Rev. 2017;05:188.

13. Statistics South Africa. Statistical release Mid-year population estimates 2016. 2016.

14. Muchengeti M, Bartels L, Olago V, et al. Cohort profile: The South African HIV Cancer Match Study (SAM) preprint. https://doi.org/10.31219/OSF.IO/ W52SB

15. Singh $E$, Ruff $P$, Babb $C$, et al. Establishment of a cancer surveillance programme: the South African experience. Lancet Oncol. 2015;16:e414-21.

16. Johnson LF, Dorrington RE, Moolla H. Progress towards the 2020 targets for HIV diagnosis and antiretroviral treatment in South Africa. South Afr J HIV Med. 2017;18:1-8

17. Fritz A, Percy C, Jack A, et al., eds. International Classification of Diseases for Oncology: Third Edition, Third. 2013 https://apps.who.int/iris/handle/ $10665 / 42344$

18. Noble M, Wright G. Using indicators of multiple deprivation to demonstrate the spatial legacy of apartheid in South Africa. Soc Indic Res. 2013:112:187-201.

19. National Department of Health. National Department of Health Data Dictionary. 2019. https://dd.dhmis.org/orgunits.html?file=NIDSIntegr ated\&source=nids\&ver=c0ed. Accessed 6 May 2021.

20. Besag J, York J, Mollie A. Bayesian image restoration with two applications in spatial statistics. Ann Inst Stat Math. 1991;43:1-20.

21. Simpson D, Rue H, Riebler A, Martins TG, Sorbye SH. Penalising model component complexity: a principled, practical approach to constructing priors. Stat Sci. 2017;32:1-28.

22. Konstantinoudis G, Schuhmacher D, Rue H, Spycher BD. Discrete versus continuous domain models for disease mapping. Spat Spatiotemporal Epidemiol. 2020;32:100319.

23. Knorr-Held L. Bayesian modelling of inseparable space-time variation in disease risk. Stat Med. 2000;19:2555-67.

24. Fuglstad GA, Simpson D, Lindgren F, Rue H. Constructing priors that penalize the complexity of gaussian random fields. J Am Stat Assoc. 2019;114:445-52

25. Gelman A, Hwang J, Vehtari A. Understanding predictive information criteria for Bayesian models. Stat Comput. 2014;24:997-1016.

26. Gómez-Rubio V, Bivand RS, Rue H. Bayesian model averaging with the integrated nested laplace approximation. Econometrics. 2020;8:23.
27. Sitas F, Newton R. Kaposi's sarcoma in South Africa. J Natl Cancer Inst Monogr. 2001;28:1-4.

28. Bray F, Colombet M, Mery L, et al. eds. Cancer Incidence in Five Continents, Vol. XI (electronic version). 2017. http://ci5.iarc.fr/CI5-XI/Default. aspx. Accessed Jun 22021

29. Rue H, Martino S, Chopin N. Approximate Bayesian inference for latent Gaussian models by using integrated nested Laplace approximations. J R Stat Soc Ser B Statistical Methodol. 2009;71:319-92.

30. Rohner E, Bütikofer L, Schmidlin K, et al. Cervical cancer risk in women living with HIV across four continents: a multicohort study. Int J Cancer. 2020;146:601-9.

31. National Cancer Registry. Cancer in South Africa 2017. 2020 https:// www.nicd.ac.za/centres/national-cancer-registry/. Accessed 6 May 2021

32. Akarolo-Anthony SN, Maso LD, Igbinoba F, Mbulaiteye SM, Adebamowo CA. Cancer burden among HIV-positive persons in Nigeria: preliminary findings from the Nigerian AIDS-cancer match study. Infect Agent Cancer. 2014;9:1.

33. Mbulaiteye SM, Katabira ET, Wabinga $H$, et al. Spectrum of cancers among HIV-infected persons in Africa: the Uganda AIDS-Cancer Registry Match Study. Int J Cancer. 2006;1 18:985-90.

34. Shiels MS, Pfeiffer RM, Gail MH, et al. Cancer burden in the HIV-infected population in the United States. J Natl Cancer Inst. 2011;103:753-62.

35. Franceschi S, Lise M, Clifford GM, et al. Changing patterns of cancer incidence in the early- and late-HAART periods: the Swiss HIV Cohort Study. Br J Cancer. 2010;103:416-22.

36. Dryden-Peterson S, Medhin H, Kebabonye-Pusoentsi M, et al. Cancer incidence following expansion of HIV treatment in Botswana. PLoS ONE. 2015:10:1-13.

37. Johnson LF, Rehle TM, Jooste S, Bekker LG. Rates of HIV testing and diagnosis in South Africa: successes and challenges. AIDS. 2015:29:1401-9.

38. Zaniewski E, Ostinelli CHD, Chammartin F, et al. Trends in CD4 and viral load testing 2005 to 2018: multi-cohort study of people living with HIV in Southern Africa. J Int AIDS Society. 2020:23:e25546.

39. Kelly $H$, Weiss HA, Benavente $Y$, et al. Association of antiretroviral therapy with high-risk human papillomavirus, cervical intraepithelial neoplasia, and invasive cervical cancer in women living with HIV: a systematic review and meta-analysis. Lancet HIV. 2018;5:e45-58.

40. Menon S, Rossi R, Kariisa M, et al. Relationship between Highly Active Antiretroviral Therapy (HAART) and human papillomavirus type 16 (HPV 16) infection among women in Sub-Saharan Africa and public health implications: a systematic review. PLoS One. 2019;14:e0213086.

41. Bratcher LF, Sahasrabuddhe W. The impact of antiretroviral therapy on HPV and cervical intraepithelial neoplasia: current evidence and directions for future research. Infect Agent Cancer. 2010;5:8.

42. Horner MJ, Altekruse SF, Zou Z, Wideroff L, Katki HA, Stinchcomb DGUS. geographic distribution of prevaccine era cervical cancer screening, incidence, stage, and mortality. Cancer Epidemiol Biomarkers Prev. 2011;20:591-9.

43. Lorenzo-Luaces Alvarez $\mathrm{P}$, Guerra-Yi ME, Faes C, Galán Alvarez Y, Molenberghs $\mathrm{G}$. Spatial analysis of breast and cervical cancer incidence in small geographical areas in Cuba, 1999-2003. Eur J Cancer Prev. 2009;18:395-403.

44. Bingi D, Gidudu A, Okello D, Mwesigwa CL. Spatial analysis of cervical cancer and correlated factors. J Remote Sens GIS. 2018;07:223.

45. Thongsak N, Chitapanarux I, Suprasert P, et al. Spatial and temporal analyses of cervical cancer patients in upper Northern Thailand. Asian Pac J Cancer Prev. 2016;17:5011-7.

46. Engels EA, Brock MV, Chen J, Hooker CM, Gillison M, Moore RD. Elevated incidence of lung cancer among HIV-infected individuals. J Clin Oncol. 2006:24:1383-8.

47. Zhao J, Virani S, Sriplung H. Spatiotemporal mapping of cervical cancer incidence highlights need for targeted prevention in Songkhla province, Thailand. Health Policy Plan. 2017;32:430-6.

48. Zhan FB, Lin Y. Racial/Ethnic, socioeconomic, and geographic disparities of cervical cancer advanced-stage diagnosis in Texas. Womens Health Issues. 2014;24:519-27.

49. Fusheini A, Eyles J. Achieving universal health coverage in South Africa through a district health system approach: conflicting ideologies of health care provision. BMC Health Serv Res. 2016:16:1-11. 
50. Ataguba JEO, Mclntyre D. Who benefits from health services in South Africa? Heal Econ Policy Law. 2013;8:21-46.

51. Spees LP, Brewster WR, Varia MA, et al. Examining urban and rural differences in how distance to care influences the initiation and completion of treatment among insured cervical cancer patients. Cancer Epidemiol Biomarkers Prev. 2019;28:882-9.

52. Matenge TG, Mash B. Barriers to accessing cervical cancer screening among HIV positive women in Kgatleng district, Botswana: a qualitative study. PLoS One. 2018;13:e0205425.

53. Akinyemiju TF, McDonald JA, Lantz PM. Health care access dimensions and cervical cancer screening in South Africa: analysis of the world health survey. BMC Public Health. 2015;15:382.

54. Simbayi LC, Zuma K, Zungu N, et al. South African National HIV Prevalence, Incidence, Behaviour and Communication Survey, 2017. Cape
Town, 2019 http://www.hsrc.ac.za/en/departments/hsc/National_HIV_ Survey.

55. Singh E, Underwood JM, Nattey C, Babb C, Sengayi M, Kellett P. South African National cancer registry: effect of withheld data from private health systems on cancer incidence estimates. South Afr Med J. 2015;105:107-9.

\section{Publisher's Note}

Springer Nature remains neutral with regard to jurisdictional claims in published maps and institutional affiliations.
Ready to submit your research? Choose BMC and benefit from:

- fast, convenient online submission

- thorough peer review by experienced researchers in your field

- rapid publication on acceptance

- support for research data, including large and complex data types

- gold Open Access which fosters wider collaboration and increased citations

- maximum visibility for your research: over $100 \mathrm{M}$ website views per year

At BMC, research is always in progress.

Learn more biomedcentral.com/submissions 